\title{
Experimental Characterization of Photonic Fiber-Integrated Modulator
}

\author{
Ran Aharoni, Moshe Sinvani, Oded Baharav, Moshe Azoulai and Zeev Zalevsky ${ }^{*}$
}

School of Engineering, Bar-Ilan University, Ramat-Gan 52900, Israel

\begin{abstract}
In this paper we present experimental characterization of a fiber-integrated, all-optical silicon based modulator. The operation principle of the proposed device is based upon plasma dispersion effect in silicon, which enables controlling the optical properties of silicon at wavelength of around $1550 \mathrm{~nm}$ by modifying the concentration of free charges either by applying external electrical field or by using illumination in spectral range that is being absorbed by this semiconductor. The concentration of the generated free charge carriers affects the real and the imaginary part of the refractive index of the silicon semi-conductor i.e. yields controllable absorption and refraction effects over the information carrier wavelength around $1550 \mathrm{~nm}$. In this paper silicon slices at varied thicknesses are integrated between an input and an output fiber and the performance of the integrated all-optical modulator are characterized versus various thicknesses of the silicon layer and the wavelength of the control signal when incident on the silicon layer from both sides or from either side of it.
\end{abstract}

Keywords: All-optical modulation, silicon photonics, plasma dispersion effect.

\section{INTRODUCTION}

The realization of fiber integrated all-optical modulators has large importance in the field of optics communication that is seeking for fast, cheap and integrated ways to perform modulation of information carried over the $1550 \mathrm{~nm}$ carrier $[1,2]$.

All-optical modulation is realized when a transmission of a certain photon is controlled by the presence of another photon. This type of modulation may achieve higher modulation rates by avoiding the conversion into electronic domain, which is much slower, noisy and cumbersome.

The most common way to control the propagation of light is by modifying the optical properties of the media in which the light propagates. This can be achieved by controlling the real and the imaginary part of the refractive index. Since Kerr effect, as mentioned in Ref. [3] and as approximated by in Ref. [4], is too small in silicon and therefore is impractical [5], the most common approach for realizing all-optical modulation in silicon is based upon the free carriers plasma dispersion effect. This was presented for the first time by Soref \& Lorenzo [6,7]. However, when used as is, the plasma dispersion effect is also weak and requires high power laser in order to achieve measurable extinction ratio of modulation.

Theoretical and experimental results involving the usage of resonators in order to overcome the optoelectronics limitations of silicon may be seen in Refs. [8,9]. Another approach which increases the interaction length by using a cylindrical lens may be seen in Ref. [10].

In general, the previously mentioned high power that is required to obtain sufficient modulation or the required large

*Address correspondence to this author at the School of Engineering, BarIlan University, Ramat-Gan 52900, Israel; Fax: 972-3-7384051; Tel: 972-35317055; E-mail: zalevsz@eng.biu.ac.il dimensions and geometries are inappropriate for effective infiber integration. However, as previously mentioned, usage of an all-fiber based configuration has advantages from the system point of view.

One interesting attempt for such an integration involved usage of a fiber having $\mathrm{Si}$ core that guides the information carrier wavelength of $1550 \mathrm{~nm}$ while a visible control beam illuminates the fiber's core from above in order to generated free carriers and to affect the absorption that is being induced over the $1550 \mathrm{~nm}$ wavelength [11]. However, this configuration is not an all-fiber or fiber-integrated one since the visible control signal is not guided through fibers and is illuminated in perpendicular to the direction of propagation of the information signal. The scheme proposed by e.g. Ref. [9] also does not include the control and the signal wavelengths to be coupled into the same fiber as done in the scheme discussed in this manuscript.

The novelty of this paper is not in the usage of the plasma dispersion effect in $\mathrm{Si}$ for generation of modulation over the $1550 \mathrm{~nm}$ carrier but rather in the construction of an all-optical modulator which is also an all-fiber or a fiber integrated module. Then, after the construction of such device we, for the first time, experimentally characterize its performance versus the control wavelength as well as versus the thickness of the integrated silicon layer.

In these experiments the information is guided within a standard optical fiber, and the control signal longitudinally illuminate the Si layer in order to imitate the case of fiber guided modulation signal (see schematic sketch in Fig. 1). The main novelty in the proposed configuration is that the control as well as the signal wavelengths are co-propagating when launched into the silicon medium. This fiber integrated scheme has advantage when used as part of optical fiber lasers for biomedical applications or when embedded into optics communication configurations. 


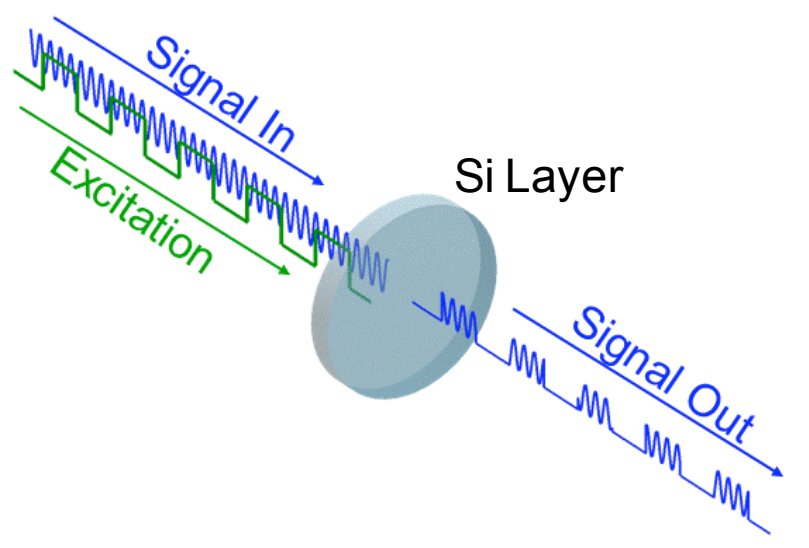

Fig. (1). Basics of a fiber-integrated provisional module. The signal (blue) can propagate freely in the medium. It is attenuated when a control pulse (green) is introduced.

In section 2 we describe the operation principle of the proposed scheme. In section 3 we present preliminary experimental results. Section 4 concludes the paper.

\section{THEORY}

The plasma dispersion effect enables optical control of optical properties of semi-conductor materials [4]. When a semi-conductor is being illuminated by photons at wavelength having energy that is higher than its band-gap, the illumination photon is being absorbed and an electron-hole pair is being generated. The concentration of those charge carriers dominate the refractive index (its real and imaginary parts) of the semi-conductor and enable modulating light at different wavelength (which is not absorbed by the semiconductor) that is being guided through the semi-conductor.

$\mathrm{Si}$ is transparent to infra-red (IR) light (having wavelength above $\sim 1.1 \mu \mathrm{m}$ ), while lower optical wavelengths are being absorbed while generating an electron-hole pair. A direct derivation from the Drude model, leads to the following equation, relating the concentration of free carriers to the generated absorption coefficient [4]:

$$
\Delta \alpha=e^{3} \lambda^{2} / 4 \pi^{2} c^{3} \varepsilon_{0} n\left[\Delta N_{e} / \mu_{e} m_{e}^{* 2}+\Delta N_{h} / \mu_{h} m_{h}^{* 2}\right]
$$

where $\Delta \alpha$ is the change in the absorption coefficient, $\lambda$ is the wavelength, $n$ is the refraction index, $\mu_{e}$ and $\mu_{h}$ are the mobility of electrons and holes respectively, $m_{e}{ }^{*}$ and $m_{h}{ }^{*}$ are the effective masses of electrons and holes respectively, $\varepsilon_{0}$ is the dielectric constant, $c$ is the speed of light in vacuum, $e$ is the charge of an electron and $\Delta N_{e}$ and $\Delta N_{h}$ are the change in the concentration of free electrons and holes in the semiconductor, respectively.

Note that the absorption coefficient is related to the imaginary part of the index of refraction:

$n=n_{R}+i \frac{\alpha \lambda}{4 \pi}$

where $n_{R}$ is the real part of the refraction index and $\alpha$ is the absorption coefficient. Using the Kramer-Kronig relations, that relate the real and imaginary parts of frequency- dependent quantities such as the linear susceptibility, one can derive the change in the real part of the refractive index:

$$
\Delta n(\omega)=\frac{c}{\pi} \cdot \wp \cdot \int_{0}^{\infty} \frac{\Delta \alpha\left(\omega^{\prime}\right) d \omega^{\prime}}{\omega^{\prime 2}-\omega^{2}}
$$

where $\wp$ is Cauchy principal value (e.g. Cauchy method for assigning values to certain improper integrals which would otherwise be undefined). This leads to:

$$
\Delta n=-e^{2} \lambda^{2} / 8 \pi^{2} c^{2} \varepsilon_{0} n\left[\Delta N_{e} / m_{e}^{*}+\Delta N_{h} / m_{h}^{*}\right]
$$

The meaning of Eqs. 1 and 4 is that illuminating Si with wavelength that is being absorbed by it, changes the index of refraction and the absorption coefficient of Si for a different wavelength (as 1550nm) for which, in normal situation, the $\mathrm{Si}$ is transparent.

The plasma dispersion effect involves several generation and recombination processes. While the generation process is a fast process (as can be seen in the experimental section), the recombination can be rather slow. Recombination can be divided into 3 main processes - (a) direct recombination, (b) recombination through traps, (c) surface recombination [12]. Since in the device described in this paper we used intrinsic $\mathrm{c}-\mathrm{Si}$, the second process is irrelevant, and due to the low charge carrier densities, Auger recombination is a negligible process as well. Some of the relevant processes are rather slow and can take up to several $\mu$ s, i.e. bulk recombination while other as surface recombination can be fast in order of several ps. For this purpose, controlling the penetration depth is of particular interest in this case since it will determine the type of recombination process being involved and thus will have a great effect on the modulation rate.

Since the penetration depth, which is defined as 1/absorption-coefficient [12], is wavelength dependent [13] especially for wavelength having energy close to the band-gap, choosing shorter wavelengths means smaller penetration. This situation gives rise to the fast surface recombination in favor of the slow bulk recombination. This assumption is valid under three conditions: the silicon is a single crystal, there are no impurities and defects (or other absorption mechanisms) that compete with the inter-band transitions, and the quantum yield is almost wavelength independent at visible and near IR wavelengths, and it is around unity, as implied by Hodgkinson [14] and Hicks et al. [15].

Several other effects take place as the wavelength is shorter - the absorption coefficient increases which causing the free charge carrier density to rise due to the shorter penetration depth, as implied by Eq. 1. As a result, and as implied by Eq. 1, the induced change in the absorption coefficient is larger as the free charge carrier increases (i.e. as the wavelength decreases) [4].

The attenuation of the signal is according to BeerLambert law: $I / I_{0}=e^{-\Delta \alpha L}$, and by substituting the first equation into it, with the appropriate penetration depths one can see that virtually, there is a dependence of the relative absorption on the wavelength and the modulation depth increases with the wavelength. The parameters in the 
simulation of Fig. (2) were taken from Soref \& Bennet [4] and from Ref. [16] while we used the free charge carrier's density that corresponds to $100 \mathrm{~mW}$ average power of the control laser (as to be specified in section 3).

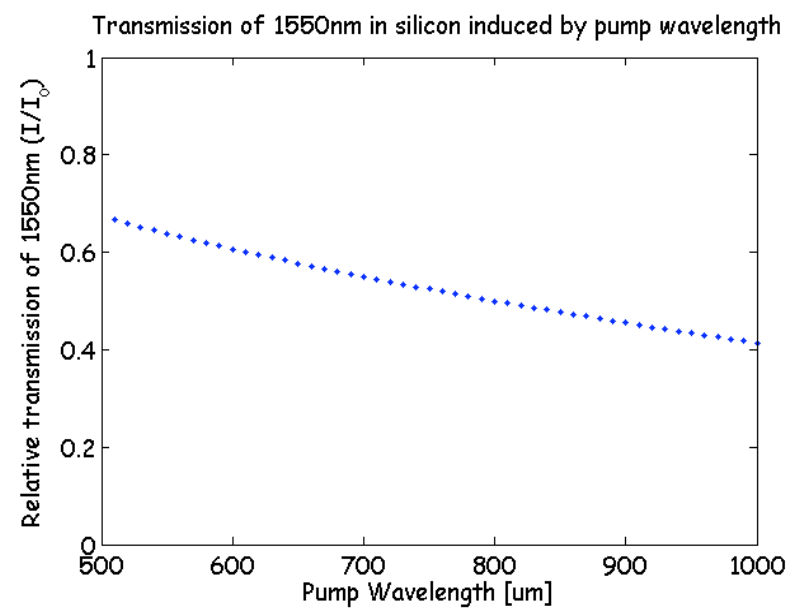

Fig. (2). Relative attenuation of $1550 \mathrm{~nm}$ signal.
Our objective, as mentioned before, is to produce a fiber integrated system, in which the signal as well as the control laser are transferred, using a fiber Y-coupler, through the same fiber. As a preliminary prototype, this fiber is to be coupled to a silicon layer through a grin-lens. The signal propagates freely in the layer and then back coupled to a fiber again, while the control signal attenuates it following the mechanism previously explained. In Fig. (3) we present part of our system where both the information as well as the control signals are coupled into fibers ending with GRIN lens to which the silicon layer is being attached. The signal laser is connected to one port of a Y-coupler through a polarization rotator and a beam splitter, and the control laser is coupled to the other port of the Y-coupler, such as the output fiber guides them both. In the following section the basics for this prototype are described, using a simplified experimental set up to simulate the situation of co-propagating signal and control lasers.

\section{EXPERIMENTAL VALIDATION}

A simplified scheme of the experimental setup is presented in Fig. (4). It includes two lasers: one at $1550 \mathrm{~nm}$, i.e.

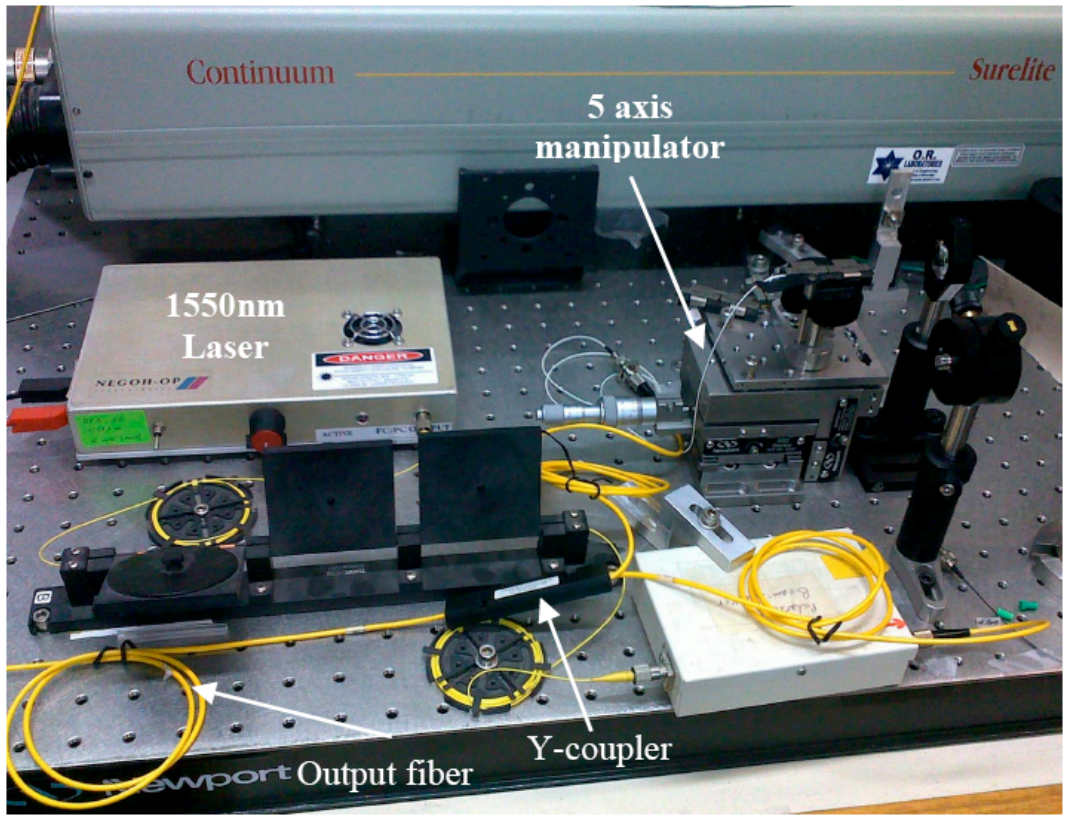

Fig. (3). Image of the experimental setup.

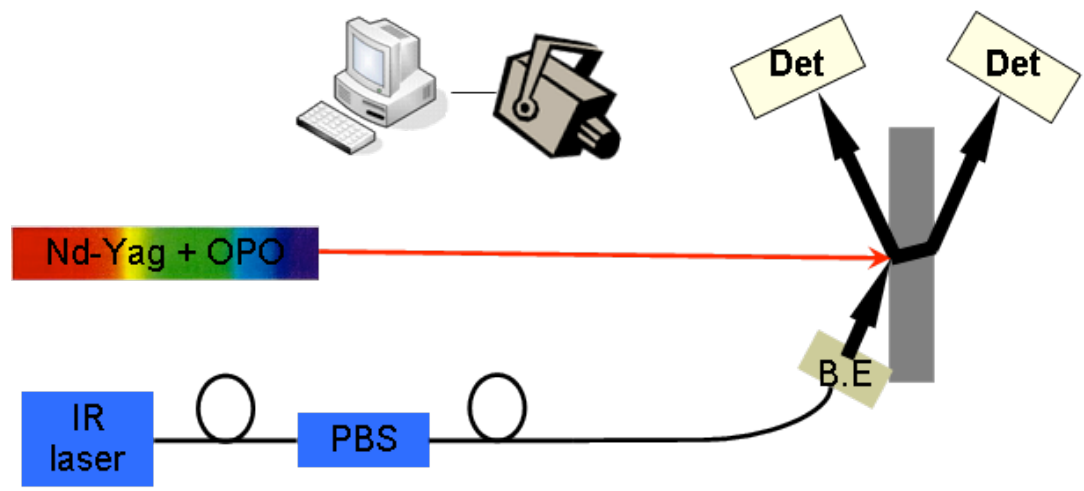

Fig. (4). Simplified scheme of the experimental system. 
the signal, and one a tunable laser, at the visible and near IR, that is used for the control. The tunable laser is a free space $\mathrm{Nd}$ :YAG pulsed laser, with an OPO generator producing $10 \mathrm{~ns}$ pulses at $20 \mathrm{~Hz}$ with varied wavelength from $350 \mathrm{~nm}$ to $2400 \mathrm{~nm}$. The signal laser is a fiber-coupled DFB diode laser. In the first set of experiments, the control signal is illuminating the silicon normally, whereas the signal is transferred through the silicon in Brewster angle to minimize losses. The detector is InGaAs/PIN with 1 ns rise time, connected to a $1.5 \mathrm{GHz} 20 \mathrm{Gs} / \mathrm{S}$ oscilloscope.

As mentioned in the last section, faster modulation rates can be achieved by relying on faster recombination mechanisms like the surface recombination, and one way of examining this idea is by using different thickness of c-Si. In the next experimentally extracted plot of Fig. (5), one can see in details that indeed using a thin layer of silicon (the sample's thickness is thin in comparison to the penetration depth which equals to $\sim 40 \mu \mathrm{m}$ for wavelength of $920 \mathrm{~nm}$ [16]) reinforces the fast surface recombination process, while in the thicker sample the bulk recombination becomes more dominant. Most of the charge carriers are near one of the two surfaces and hence recombine faster while the remaining carriers recombine in the bulk. The process gets slower as the charge carriers density drops, and the diffusion process from the bulk to the surface becomes negligible.

Recombination processes in $3 \mathrm{~mm}$ and $20 \mu \mathrm{m}$ silicon $920 \mathrm{~nm}$

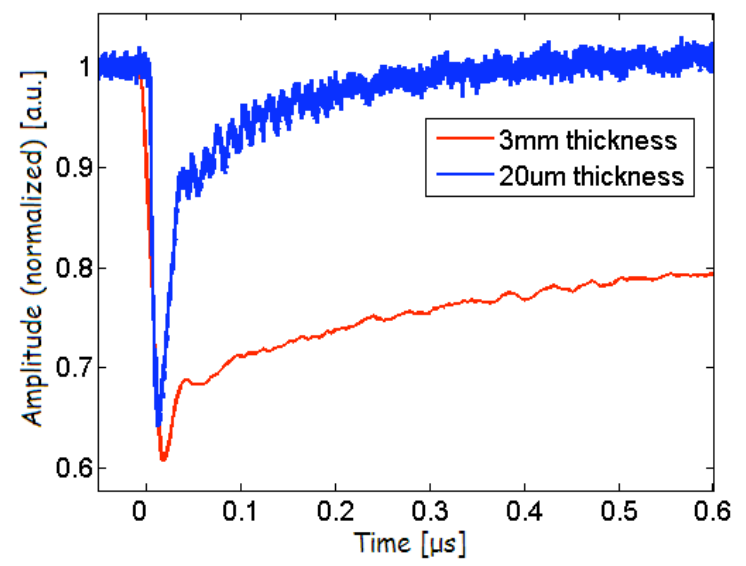

Fig. (5). Comparison of the temporal response obtained at $20 \mu \mathrm{m}$ thick (blue) and $3 \mathrm{~mm}$ thick (red) c-Si samples when being illuminated with a control pulse of $10 \mathrm{nsec}$ at wavelength of $920 \mathrm{~nm}$. The bulk and surface recombination can easily be distinguished.

Fig. (5) shows quite similar attenuation for both samples, although the thin layer of silicon $(\sim 20 \mu \mathrm{m})$ is thinner than the penetration depth $(\sim 40 \mu \mathrm{m})$. Let us compare this case to a sample as thick as the penetration depth: since the control laser decays exponentially with the distance, in $40 \mu \mathrm{m} 63 \%$ of the radiation is absorbed while in $20 \mu \mathrm{m} 40 \%$ is absorbed. The remaining $60 \%$ suffers back reflection from the siliconair interface and more than $30 \%$ of it is reflected back into the sample, and more than $60 \%$ of the radiation is absorbed in the $20 \mu \mathrm{m}$ sample (in comparison to a $40 \mu \mathrm{m}$ sample).

In addition, in this figure the data is not normalized to the control laser pulse energy which has a stability of a few percents, and there is a difference between the surface quality of the samples.

Another approach to produce faster modulation rates is to use shorter wavelengths that reinforce surface recombination process due to its shorter penetration depth. In Fig. (6) one can see the experimental results of the response of signal at wavelength of $1550 \mathrm{~nm}$ to various control wavelengths. A significant increase in the attenuation with the wavelength is noticed, which is smaller from the dependence we mentioned before (see Fig. 2).

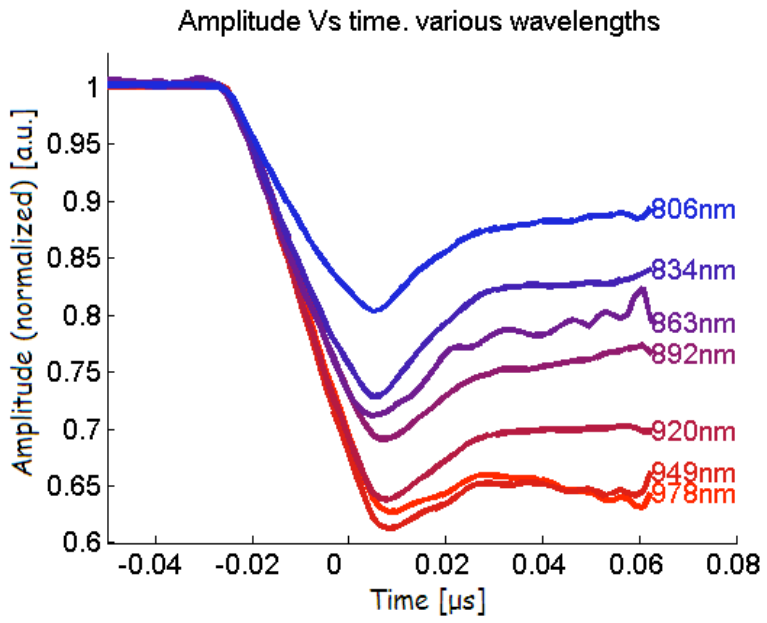

Fig. (6). Influence of control laser wavelength on the recovery time of the signal laser.

There are several reasons to that dependence: first, for a fixed laser power the number of photons is changing as the energy is wavelength dependent: $E_{p h}=h v$ where $h$ is Plank constant, $c$ the speed of light and $v$ the frequency. The total energy carried in a pulse equals to $E_{t}=N_{p h} \cdot E_{p h}=N_{p h} \cdot h c / \lambda$ where $N_{p h}$ is the number of photons. Thus, as the wavelength increases the number of photons increases (and the reflection decreases as well), and the plasma dispersion effect is becoming stronger. Another reason is that at higher charge carriers density (i.e. shorter wavelengths) the diffusion is stronger, and charge carriers reduce their density by faster depletion both laterally as well as into the bulk. In addition, at small wavelengths the surface recombination is enhanced and so does the Auger recombination. These processes can be orders of magnitude faster than other mentioned processes. Also from the pulse temporal duration point of view some generated electron-hole pairs recombine faster than we can observe in our experiment and by that they are reducing the carriers population.

In fiber integrated modulators except of fast response rate it is important also to achieve high extinction ratio for the modulation process. The problem is that increasing the average power of the excitation wavelength at a given power stops increasing the extinction ratio since the high concentration of the free carriers acts as a mirror which prevents from additional excitation photons to penetrate into the silicon layer. In Fig. (7) we present experimental measure- 
ments showing that indeed the extinction ration reaches an asymptote (saturation). The diameter of the beam spot illuminating the Si layer in this experiment was about $2 \mathrm{~mm}$ (verified by measurement) for both the excitation as well as the information wavelengths.

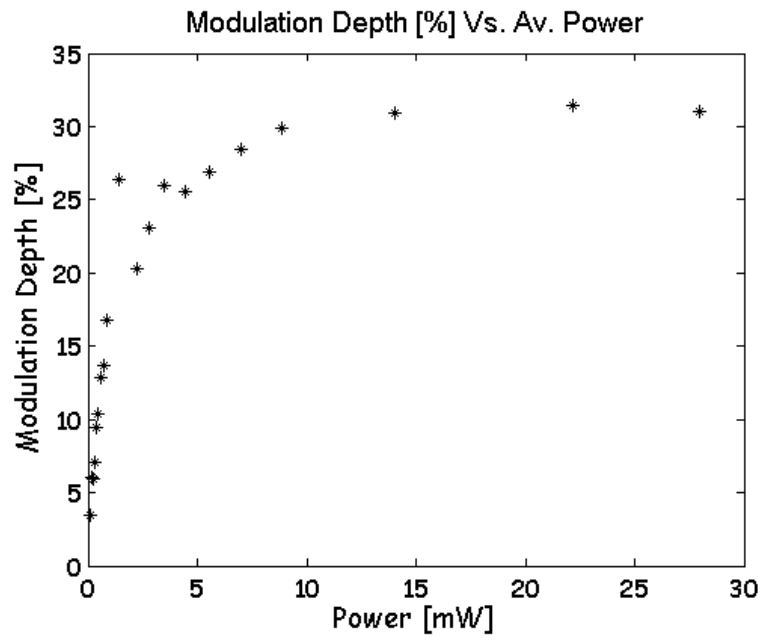

Fig. (7). Experimental results demonstrating that increasing the average power of the excitation photons cannot further enhance the extinction ratio.

In order to emphasize the fast surface recombination effect, and in order to produce a fast device with a high extinction ratio, we used the Nd:YAG second harmony $(532 \mathrm{~nm})$, having penetration depth of only about $1 \mu \mathrm{m}$ which suppresses the bulk effect. In addition, we enhanced the total absorption by using the tandem effect - we illuminated the sample from both sides (instead of just increasing the power of the excitation photons for having higher extinction ratio). This procedure almost doubles the total absorption, and kept the high speed of operation due to the surface effect. The used experimental setup is presented in Fig. (8).

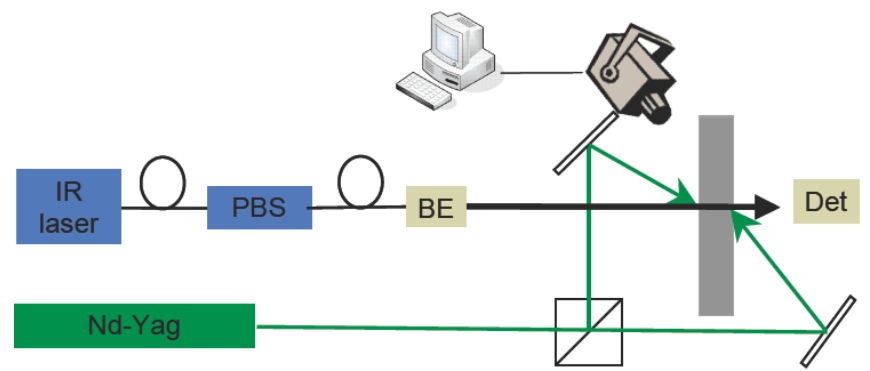

Fig. (8). The experimental setup.

In the plot of Fig. (9) we presented the effect of illumination on both sides of the silicon layer in comparison to single side illumination. One can see that the tandem modulation depth (green) not equals to the sum of the backside and front side illumination separately as the attenuation is relative. The extinction ratio can further be enhanced if instead of a double pass a multiple pass through the Si layer is generated. This can be done when the Si layer is integrated between two reflective surfaces such that a Fabry-Perot resonator is realized.

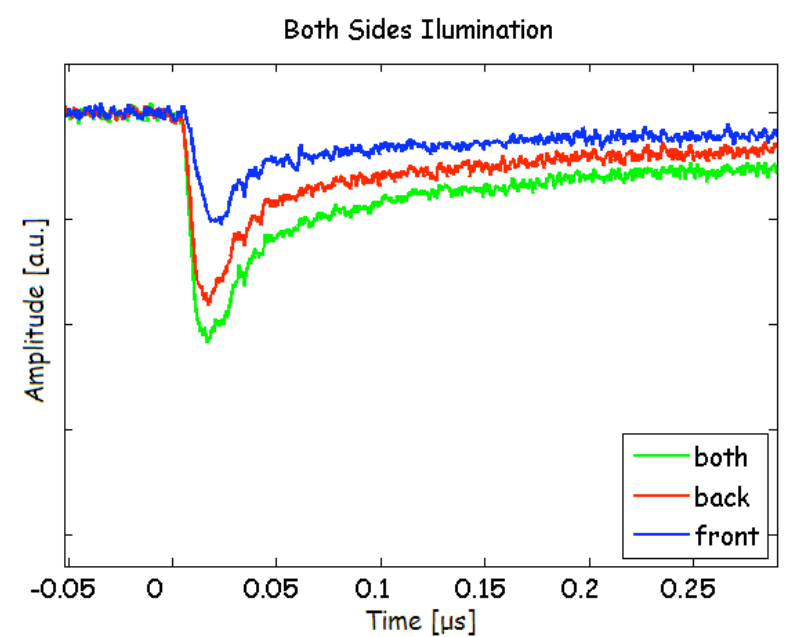

Fig. (9). Experimental results comparing illumination of the control signal on both sides of the silicon layer versus illumination applied on each one of the sides separately.

\section{CONCLUSIONS}

In this paper we have constructed the basics of a fiberintegrated provisional module, by realizing an all-optical modulator. In this configuration, the control and signal wavelengths are co-propagating and then subsequently launched onto a silicon sample.

Control laser wavelength consideration have been discussed, and we showed correspondence between the experimental values and the theoretical effect of the excitation wavelength on the overall attenuation. We also showed the relation between the control wavelength and the modulation rate.

Preliminary experimental results demonstrated the proposed operation principle when the control laser light incident on the silicon layer from both sides or from either side of it.

Although the presented system is an evaluation set, the demonstrated principle validates the feasibility of the next research step in which a thin silicon layer will be integrated into a Fabry-Perot resonator realized between two single mode fibers.

\section{REFERENCES}

[1] Soref RA. Silicon-based optoelectronics. Proc IEEE 1993; 81: 1687-706.

[2] Alferness RC. Guided wave devices for optical communication. IEEE J Quantum Electron 1981; QE-17: 946-58.

[3] Moss TS, Burrell GJ, Ellis B. Semiconductor opto-electronics. London. Butterworths; 1975.

[4] Soref RA, Bennett B. Electrooptical effects in silicon. IEEE J Quantum Electron 1987; QE-23: 123-9.

[5] Dinu M, Quochi F, Garcia H. Third-order nonlinearities in silicon at telecom wavelengths. Appl Phys Lett 2003; 82: 2954-6.

[6] Soref RA, Lorenzo JP. All-silicon active and passive guided-wave components for $\lambda=1.3$ and $1.6 \mu \mathrm{m}$. IEEE J Quantum Electron 1986; QE-22: 873-9. 
[7] Lorenzo JP, Soref RA. $1.3 \mu \mathrm{m}$ electro-optic silicon switch. Appl Phys Lett 1987; 51: 6-8.

[8] Manolatou C, Lipson M. All-optical silicon modulators based on carrier injection by two-photon absorption. J Lightwave Technol 2006; 24: 1433-9.

[9] Almeida VR, Barrios CA, Panepucci RR, Lipson M. All-optical control of light on a silicon chip. Nature 2004; 431: 1081-3.

[10] Stepanov S, Ruschin S. Modulation of light by light in silicon-oninsulator waveguides. Appl Phys Lett 2003; 83: 5151-3.

[11] Won DJ, Ramirez MO, Kang H, et al. All-optical modulation of laser light in amorphous silicon-filled microstructured optical fibers. Appl Phys Lett 2007; 91: 161112.
[12] Smith RA, Semiconductors: 2nd ed. Cambridge University Press, Cambridge, 1978.

[13] Schroder DK, Thomas RN, Swartz JC. Free Carrier Absorption in Silicon. IEEE J Solid-State Circ 1978; 13(1): 180-7.

[14] Hodgkinson RJ. Impact ionization and quantum efficiency in silicon. Proc Phys Soc 1963; 82(1): 58.

[15] Hicks C, Kalatsky M, Metzler R, Goushcha A. Quantum efficiency of silicon photodiodes in the near-infrared spectral range. Appl Opt 2003; 42: 4415-22.

[16] Green MA, Keevers MJ. "Optical properties of intrinsic silicon at 300 K. Progress in Photovoltaics; Res Appl 1995; 3: 189-92.

(C) Aharoni et al.; Licensee Bentham Open.

This is an open access article licensed under the terms of the Creative Commons Attribution Non-Commercial License (http://creativecommons.org/ licenses/by-nc/3.0/), which permits unrestricted, non-commercial use, distribution and reproduction in any medium, provided the work is properly cited. 